\title{
Authoring Tool for Location-Aware Experiences
}

\author{
Federico M. Alconada Verzini \\ LIFIA, Fac. de Informática, UNLP. \\ Calle 50 y 120, La Plata, \\ Buenos Aires, Argentina. \\ +54 $221422-8252$ \\ falconada@lifia.info.unlp.edu.ar \\ Juan I. Tonelli \\ LIFIA, Fac. de Informática, UNLP. \\ Calle 50 y 120, La Plata, \\ Buenos Aires, Argentina. \\ +54 $221422-8252$ \\ jtonell@lifia.info.unlp.edu.ar
}

\author{
Cecilia Challiol $^{\ddagger}$ \\ LIFIA, Fac. de Informática, UNLP. \\ Calle 50 y 120, La Plata, \\ Buenos Aires, Argentina. \\ +54 $221422-8252$ \\ ceciliac@lifia.info.unlp.edu.ar
}

\author{
Alejandra B. Lliteras \\ LIFIA, Fac. de Informática, UNLP. \\ Calle 50 y 120, La Plata, \\ Buenos Aires, Argentina. \\ +54 $221422-8252$ \\ Iliteras@lifia.info.unlp.edu.ar
}

\author{
Silvia E. Gordillo§ \\ LIFIA, Fac. de Informática, UNLP. \\ Calle 50 y 120, La Plata, \\ Buenos Aires, Argentina. \\ +54 $221422-8252$ \\ gordillo@lifia.info.unlp.edu.ar
}

\begin{abstract}
In this paper, we present an approach to create location-aware experiences. We use the concept of separation of concerns to represent the content layer and the location layer. This separation allows reusing the layers independently one of each other. The focus of this paper is to provide an end-user tool to create location-aware applications in-situ. By using our tool, the location-aware experiences can be defined in both indoor and outdoor spaces. We present an example of how our tool is used and we describe some discussion points that have occurred to us while defining a tool with these characteristics.
\end{abstract}

\section{Categories and Subject Descriptors}

D.2.2 [Software Engineering]: Design Tools and Techniques Object-oriented design methods, User interfaces.

H.5.2 [Information Interfaces and Presentation]: User Interfaces - User-centered design

\section{General Terms}

Design

\section{Keywords}

In-situ Authoring Tool; Location-Aware Experiences; IndoorOutdoor Space; Separation of Concern; Mobile Applications

\section{INTRODUCTION}

The increasing capabilities of mobile devices (e.g. GPS, NFC, etc.) are leading to a new way of creating mobile applications in

\footnotetext{
$\ddagger$ Another affiliation for Cecilia Challiol: CONICET, Argentina.

$\S$ Another affiliation for Silvia E. Gordillo: CIC, Buenos Aires, Argentina.
}

Permission to make digital or hard copies of all or part of this work for personal or classroom use is granted without fee provided that copies are not made or distributed for profit or commercial advantage and that copies bear this notice and the full citation on the first page. To copy otherwise, or republish, to post on servers or to redistribute to lists, requires prior specific permission and/or a fee.

NHT'15, September 1, 2015, Guzelyurt, Northern Cyprus.

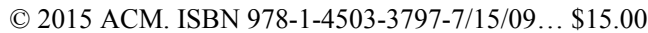

DOI: http://dx.doi.org/10.1145/2804565.2804570 which end-users are getting involved. Nowadays, users not only create contents (e.g. taking pictures, creating comments, etc.) but also mobile applications; in particular Location-Aware applications [13].

Location-Aware applications can be used for different purposes and no matter which domain they have, the user of the application receives information based on his physical location. These domains can vary, going from education to games (more examples are mentioned in [7]).

Up until the last few years this kind of applications has been being created by developers. But this has changed with the growing use use of mobile devices and users have become part of these creations.

According to [12], end-users have two different ways to design location-aware applications: digital or physical. In the first case (digital design), a map is used as a reference to define the relevant locations and contents but users are not physically located there. For example, [10] and [12] approaches provide authoring tools that use maps to define relevant locations (and its associated content). In the second case (physical design), the user is physically located in the place where the relevant locations and contents will be defined. In the literature, this last way of designing is generally known as "in-situ creation" ([4] and [9]). As mentioned in [11] and [13], being in-situ enables users to identify relevant environmental aspects of the real world. This can be important not only to choose a more suitable location but also to define a more accurate content associated with each place.

In general, in-situ tools create a content coupled with a location and are only focused on outdoor applications. For example, CASTOR [9] associates a GPS location with each content. Another similar example is TOTEM [4], which allows choosing a GPS or an NFC location. Both approaches provide support to create only outdoor mobile applications.

In this paper, we present an authoring tool to create locationaware applications not only for outdoor spaces but also for indoor ones. This tool has been defined in a generic way so it can be used for any domain (education, entertainment, etc.). In addition, we have focused on providing a solution to decouple contents 
from locations by using the concept of separation of concerns which we have been analysing and modelling for mobile applications in our previous works ([1] and [2]). Furthermore, we present an example of how our tool is used and we describe some issues that have sprung to our minds while defining a tool with these characteristics.

This paper is organised as follows. In Sections 2 we describe some related works. Our approach is presented in Section 3. Our authoring tool is shown in Section 4. Conclusions and future work are mentioned in Section 5.

\section{RELATED WORK}

In [3], methods and tools for producing location-based Mobile Urban Dramas are presented. In this kind of applications, users become the main characters in a play. The authors define a framework to create these dramas. In particular, the authors present six dramas with different purposes, for example, tourism and learning. Note that these dramas are created by developers.

Structuring location-aware narratives is described in [7]. The authors present three patterns characterised using [5] and, based on this, they provide a sculptural location-based hypertext model. This model defines the concept of location-query as an attribute of different classes. Until now, this approach is focused on modelling aspects of these kinds of applications and not to provide an end-user tool.

As it was previously mentioned, end-users can design locationaware applications in two ways, digital or physical [12]. The former uses a map as a reference to define the relevant locations and contents but users are not physically located there. The latter, requires the user to be physically located in the place in which the application will be used later. Concrete examples of both approaches are described next.

In [12], a digital design tool called QuesTInSitu is presented. This tool enables users to define learning activities in specific outdoor locations. The activities defined can be run (by a student) using the mobile web application's user interface. The tool provides a map that uses a web map service (e.g. Google Maps), which is used by teachers, to create in-situ activities in relevant geolocations. The concept of in-situ activities means that, when the students reach one of these particular geolocations (using their mobile device's GPS), they receive the corresponding activity that is automatically triggered by the tool.

Another digital design is presented in [10]. The authors describe the IVO platform, which allows end-users to build and deploy mobile context-aware applications. To support this kind of applications, IVO platform uses an event-driven model. As part of the IVO platform, the authors also provide the IVO Builder and the IVO Client (Android application). In [10] is shown a tourist guide application created by using this platform.

Regarding to the physical design, two approaches are presented. In [9] the author describes CASTOR, (Context Aware STORytelling) an authoring system oriented to children that enables them to create structured stories in-situ. This system supports structuring the content both with a sequential logic or using the concept of branching. This system uses the mobile device's GPS to retrieve a specific geolocation that is associated to different contents (text, pictures, etc.). Moreover, in each location students specify contextual conditions used to trigger the content when the final mobile application is running.
The other similar in-situ authoring tool is presented in [4]. This is an Android application called TOTEM.Scout that allows users to create location-based content (image, video, etc.). The authors provide the concept of Shapes that are templates users can use to define contents (associated to relevant locations). These templates provide, for example, the possibility to associate a GPS or NFC location with the content. In addition, the authors provide the TOTEM.Design (a web-based tool) that uses the created content to build location-aware experiences. Up to now, the authors only support Google Maps and they mentioned as the future work to add floor plants to their tool.

In conclusion, all these mentioned approaches are focused on the production of different kinds of outdoor mobile applications. Additionally, the contents defined on these approaches are coupled with a specific location (generally a GPS location). Taking into account the contribution of this paper, we present a tool to define indoor-outdoor location-aware experiences, which models contents and locations in a decoupled way (providing reusability of both contents and locations).

\section{OUR APPROACH}

In Figure 1, a general schema of our approach is shown. We provide a responsive web-based interface used to define locationaware experiences. Our framework is used to provide the functionality required to build an experience (Figure 1 shows that the framework returns a final mobile experience). Web Services are used to communicate both parts (interface and framework). Our interface is capable of communicating both with map services (e.g. for outdoor representations) and sensing mechanisms such as GPS.

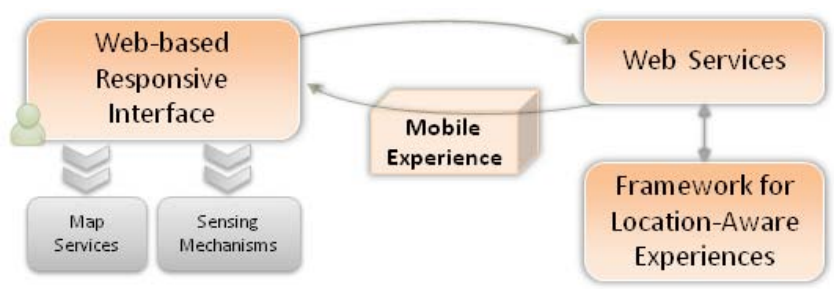

Figure 1. General schema of our approach.

Our approach defines general concepts used by our end-user tool and framework. We have identified two different layers (or concerns), one with contents (text, image, video, multiple choice question, etc.) and its relationships, and the other one that defines relevant locations (related with an indoor or outdoor space). Locations can be defined in different ways, such as in a symbolic or geometric way, as mentioned in [6]. The relationship between contents and locations define a point of interest (PoI). These are shown in the Figure 2.

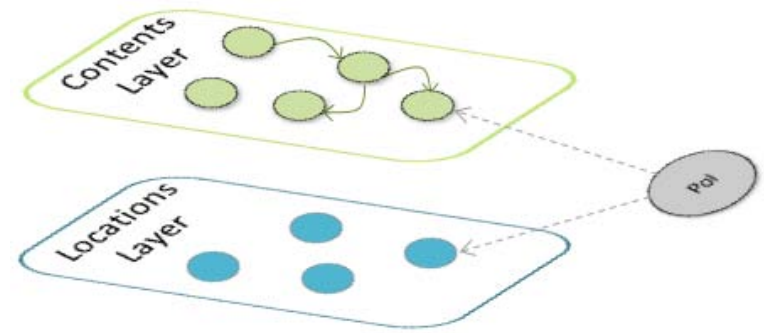

Figure 2. General concepts used in our approach. 
Using the concepts defined in Figure 2, contents and locations can be part of different PoI, allowing reuse. This can be interesting and useful for different situations. For instance, if a museum used QR-codes associated to each object with our approach, the location layer could be reused to provide different location-aware experiences. Each experience could define different contents (related with their locations) with different purposes, such as learning activities or tour guides.

\section{AUTHORING TOOL}

In this section, we present our visual tool to create location-aware experiences. This tool is defined by using a responsive web-based interface so it can be used in any web browser (mobile or desktop). Up to now, our tool provides two different ways of defining locations, using GPS or QR-code. But its flexible design supports the addition of any other location sensing mechanism. Our tool enables users to define:

- Contents Layers

- Locations Layers (relative to an indoor or outdoor space)

- PoI (a content within a location forms a point of interest)

In this paper, we only focus on the creation of PoI. To obtain the location, a user can configure the location sensing mechanism. By default, GPS is chosen but he can switch into QR-codes whenever he wants. In the case of GPS, the behaviour is similar to the related work presented in Section 2. If QR-codes are used, a new code is generated for each content (in a future approach of the tool, we will consider the possibility of reading existing QRcodes). Each code is related to an indoor-outdoor location inside the tool's map. In the final application, QR-codes are used to obtain the contents associated to them. Either using GPS or QRcodes we create a separation layer with these locations.

In the following sections, we show an example of the usage of our tool and some discussion points.

\subsection{Using our tool}

A first version of our tool is presented in this section. The tool supports defining PoI in an indoor, outdoor or a mixed space (indoor-outdoor). The most interesting example is the latter, as it uses both indoor and outdoor spatial representations. In this first version, we have decided to use an image of the indoor space (the museum's plan) to represent the indoor space. Figure 3 shows how the image is overlaid over the outdoor map.

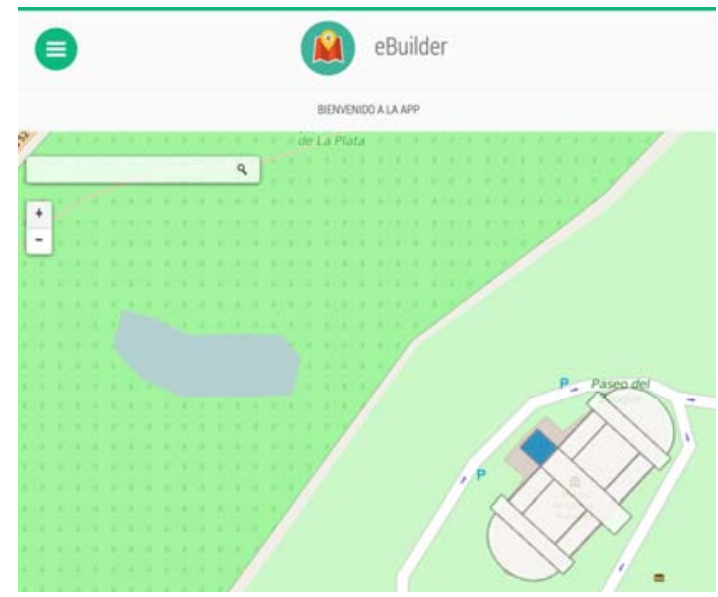

Figure 3. Indoor-Outdoor Map using our tool.
In Figure 4, the different types of content provided by our tool are shown, each one with one icon.

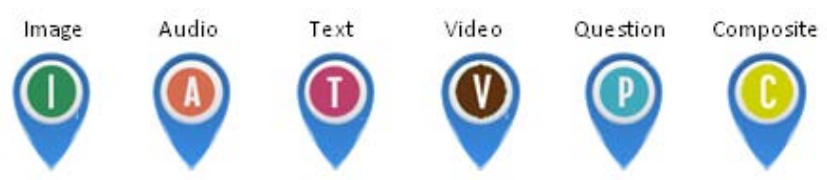

Figure 4. Icons representing contents in our tool.

Suppose a user is interested in creating a guided tour showing different mammal animals. In La Plata city (Argentina), the Zoo (outdoor) and the Natural Science Museum (indoor) are very close. The user wants to show some extinct animals (exhibited in the Museum) and some non-extinct animals (which are in the Zoo).

Using our tool the user can visit the Zoo and the Museum, and in each place define PoI. At the Zoo, the user takes advantage of the GPS sensing mechanism to create the different PoI. As he walks through the different places, he creates the different PoI and the markers representing each one of them are automatically placed on his/her current position.

Figure 5 shows some PoI created in the outdoor space (the zoo) and a linear relationship between them. For simplicity, assume that for each PoI only a text is defined.

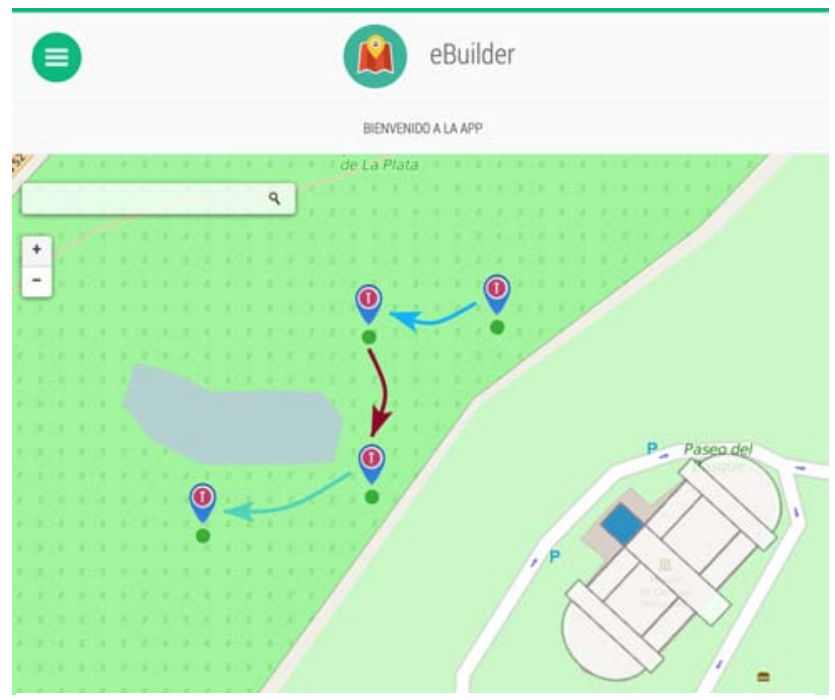

Figure 5. PoI defined in the outdoor map.

When the user is inside the Museum, he decides to switch to the QR-code sensing mechanism. As in this case there is no GPS coordinate representing the user's current position, the tool randomly places the PoI anywhere in the map. And this is why the user must manually drag the markers to the correct places on the tool's map. Every time the user creates a new PoI, a QR-code is generated as shown in Figure 6. 


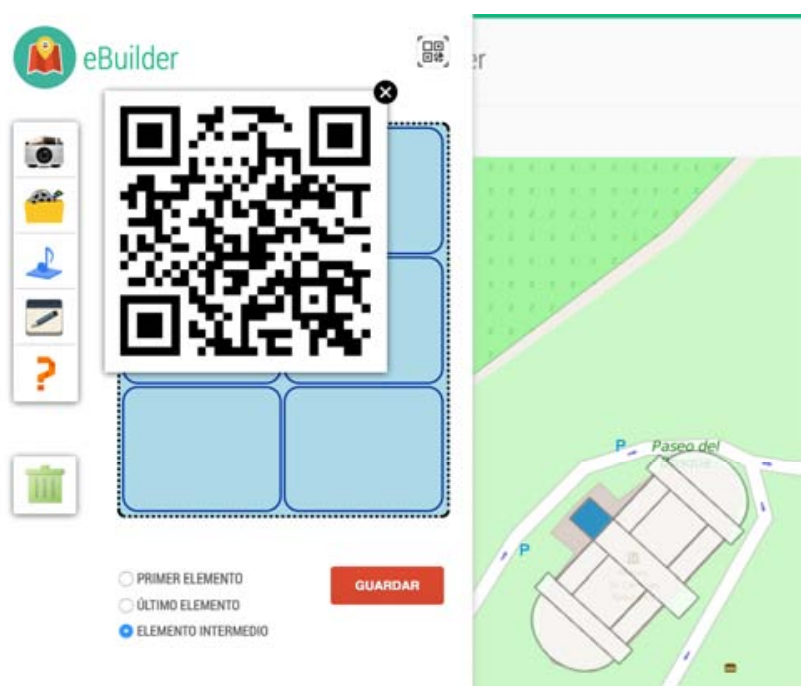

Figure 6. QR-code associated to a PoI.

Figure 7 shows all the PoI created by the user. Note that one of the outdoor PoI is connected with one of the indoor PoI

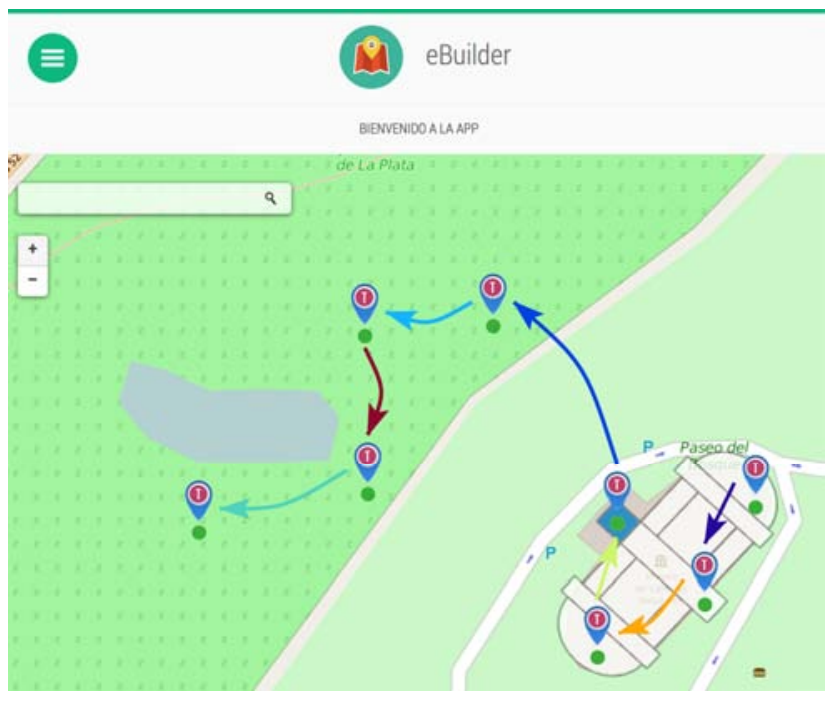

Figure 7. An indoor-outdoor tour defined with our tool.

As part of the final application, all QR-codes (if there are) are given to the user, who will be in charge of placing those codes in the correct physical locations. The framework builds the experiences as web applications with the possibility of using Phonegap [8] to create hybrid applications.

\subsection{Discussions}

Nowadays, most existing authoring tools do not provide any location sensing mechanism for indoor spaces. Consequently, not having this location assistance is the reason why creating mobile applications by end-users is more complex. The use of QR-codes is a first approach to define relevant locations. But this also depends on settling that code in the correct place.

The indoor representation is a topic by itself. In our first version of the tool, we only consider a referenced image to simplify it.
But we know that this is not scalable in order to build an indoor space with more than one floor. In these cases, the tool will should be defined with a more complex representation as shown in [14].

The complexity of the tool increases when context features are defined as part of each PoI, as presented in [9]. Up to now, the first version of our tool only allows defining simple contents such as: text, figure, audio, video, multiple-choice questions or a composite of them.

Another interesting point to mention is the layer's reusability. In this case, location layer's reusability is possible because they just define relevant locations in an indoor-outdoor space. But the reuse of contents is not always possible if it is location-dependent (as is presented in [13]). In other words, if the content mentions any characteristic of the environment, the reuse is limited.

It is important to note that, with our tool, it is possible to define the three structure patterns mentioned in [7]. These structures are dynamically defined according to the relationships that users define between the contents.

\section{CONCLUSION AND FUTURE WORK}

We have presented an approach to create location-aware experiences. As mentioned before, we have used the concept of separation of concerns to represent the content layer and the location layer. To relate content and location, we have used the concept of PoI. We have presented a first version of our tool, which creates location-aware applications in-situ. Our tool uses indoor-outdoor spaces to define $\mathrm{PoI}$ and relationships between them. These are shown in the example presented in this paper. Some discussion points have been presented to show that this is the starting point of an area that requires much more research and evaluation.

Up to the moment, the tool has only been evaluated by the developers involved in its definition. We know that it is crucial to evaluate it with end-users. To achieve this, we are currently organising an experiment to create a specific location-aware application that involves indoor-outdoor places to be evaluated by end-users.

We are also working on providing more complex indoor representations as it is defined in [14]. According to that, we have evaluated different kinds of interfaces to ease the definition of PoI when the indoor space has more than one floor.

As future work, the tool will support more sensing mechanisms such as NFC. Furthermore, the tool will define more complex contents. For example, users will be able to record videos or take pictures (as is described in [4]).

\section{REFERENCES}

[1] Fortier, A., Challiol, C., Fernández, J. L., Robles, S., Rossi, G., and Gordillo, S. 2014. Exploiting personal web servers for mobile context-aware applications. The Knowledge Engineering Review 29, 2, (Mar. 2014), 134-153. DOI=http://dx.doi.org/10.1017/S0269888914000022

[2] Fortier, A., Rossi, G., Gordillo, S. E., and Challiol, C. 2010. Dealing with variability in context-aware mobile software. Journal of Systems and Software83, 6, (June 2010) 915-936. $\mathrm{DOI}=$

http://www.sciencedirect.com/science/article/pii/S01641212 09002830 
[3] Hansen, F. A., Kortbek, K. J., and Grønbæk, K. 2012. Mobile urban drama: interactive storytelling in real world environments. New Review of Hypermedia and Multimedia18, 1-2, (Mar.-June 2012), 63-89. DOI= http://www.tandfonline.com/doi/abs/10.1080/13614568.2012 .617842

[4] Jurgelionis, A., Wetzel, R., Blum, L., and Oppermann, L. 2013.TOTEM.Scout: A mobile tool for in-situ creation of location-based content. In Proceedings of Games Innovation Conference 2013 (Vancouver, BC, September 23-25, 2013). IGIC 2013. IEEE, 89-96.

[5] Kjeldskov, J., and Paay, J. 2007. Augmenting the City with Fiction: Fictional Requirements for Mobile Guides. In Proceedings of Mobile Interaction with the Real World 2007/5th Workshop on HCI in Mobile Guides (Singapore, September 9, 2007). MIRW 2007. ACM, New York, NY, 16.

[6] Leonhardt, U. 1998. Supporting location-awareness in open distributed systems. Doctoral dissertation, Imperial College.

[7] Millard, D. E., Hargood, C., Jewell, M. O., and Weal, M. J. 2013. Canyons, deltas and plains: towards a unified sculptural model of location-based hypertext. In Proceedings of the 24th ACM Conference on Hypertext and Social Media (Paris, France, May 01-03, 2013). Hypertext 2013. ACM, New York, NY, 109-118. DOI= http://dl.acm.org/citation.cfm?doid=2481492.2481504

[8] Phonegap Home Page: http://phonegap.com/

[9] Pittarello, F., and Bertani, L. 2012. CASTOR: learning to create context-sensitive and emotionally engaging narrations in-situ. In Proceedings of the 11th International Conference on Interaction Design and Children (Bremen, Germany, June 12-15, 2012). IDC 2012. ACM, New York, NY, 1-10. DOI= http://dl.acm.org/citation.cfm?doid=2307096.2307098
[10] Realinho, V., Romão, T., Birra, F., \& Dias, A. E. 2011.Building mobile context-aware applications for leisure and entertainment. In Proceedings of the 8th International Conference on Advances in Computer Entertainment Technology (Lisbon, Portugal, 8-11 November, 2011). ACE 2006. ACM, New York, NY, Article No. 29.

DOI=http://dl.acm.org/citation.cfm?doid=2071423.2071459

[11] Rogers, Y., Connelly, K., Tedesco, L., Hazlewood, W., Kurtz, A., Hall, R. E., and Toscos, T. 2007. Why it's worth the hassle: The value of in-situ studies when designing ubicomp. In Proceeding of the 9th international conference on Ubiquitous Computing (Innsbruck, Austria, September 16-19, 2007). UbiComp 2007. Springer Berlin Heidelberg, 336-353.

[12] Santos, P., Hernández-Leo, D., and Blat, J. 2014. To be or not to be in situ outdoors, and other implications for design and implementation, in geolocated mobile learning. Pervasive and Mobile Computing 14, (Oct. 2014), 17-30.

[13] Weal, M. J., Hornecker, E., Cruickshank, D. G., Michaelides, D. T., Millard, D. E., Halloran, J., and Fitzpatrick, G. 2006. Requirements for in-situ authoring of location based experiences. In Proceedings of the 8th conference on Human-computer interaction with mobile devices and services (Helsinki, Finland, September 12-15, 2006). Mobile HCI 2006. ACM, New York, NY, 121-128. DOI= http://dl.acm.org/citation.cfm?doid=1152215.1152241

[14] Zlatanova, S., Liu, L., and Sithole, G. 2013. A conceptual framework of space subdivision for indoor navigation. In Proceedings of the Fifth ACM SIGSPATIAL International Workshop on Indoor Spatial Awareness (Orlando, Florida, USA, November 5-8, 2013). ISA 2013. ACM, New York, NY, 37-41. DOI= http://dl.acm.org/citation.cfm?doid=2533810.2533819 